\title{
Verification of a 2D finite element debris flow model using Bingham and cross rheological formulations
}

\author{
C. Martinez ${ }^{1}$, F. Miralles-Wilhelm ${ }^{1} \&$ R. Garcia-Martinez ${ }^{2}$ \\ ${ }^{I}$ Department of Civil and Environmental Engineering, \\ Florida International University, Miami, USA \\ ${ }^{2}$ Applied Research Center, Florida International University, \\ Miami, USA
}

\begin{abstract}
We propose a vertically-averaged 2D debris flow model based on the nonNewtonian Bingham and Cross rheological formulations. In the Cross model, fluid viscosity changes continuously through the range of shear rates. The $2 \mathrm{D}$ model is based on the shallow water equations, where the internal friction losses are implemented using the Cross constitutive relations. The numerical method is based on a four-step, selective lumping, explicit time stepping scheme. We present preliminary tests for one dimensional and two dimensional dam break problems. Model results show very good agreement with experimental data and analytical solutions in one dimensional cases. For the flow early stages, the numerical solution agrees better with the experimental data than with the analytical solution. However, in final stages, the numerical solution predicts that the fluid front advances more rapidly than the analytical solution and measured fluid front. The proposed 2D debris flow model provides very stable results even in the range of very low shear rates, where other discontinuous constitutive relations that use the yield stress may become unstable.

Keywords: debris flow, Cross model, Bingham model, yield stress, finite elements.
\end{abstract}




\section{Introduction}

Mud and debris flows can be characterized as a flowing sediment-water mixture driven by gravity. Rheological studies of mud at high enough solid concentrations have shown that it behaves as a very viscous, non-Newtonian fluid, exhibiting a yield stress as evidenced by an minimum depth needed for a uniform layer of mud to flow. Various rheological models have been proposed $[2,3,5,10]$, being the Bingham plastic model the most commonly used in practice. In simple shear, the stress and shear rate relation for a Bingham fluid is:

$$
\tau=\left\{\begin{array}{c}
0 \quad \text { if } \quad \tau \prec \tau_{y} \\
\tau_{y}+\mu \dot{\gamma} \quad \text { if } \quad \tau \geq \tau_{y}
\end{array}\right.
$$

where $\tau$ is the shear stress, $\tau$ is the yield stress, $\mu$ is the dynamic viscosity and $\dot{\gamma}$ is the shear rate. Many authors have proposed the Bingham rheological model to simulate debris flow. However, the hypothesis of a critical or yield shear stress may create instabilities in some model applications since, for close to zero shear rates, the Bingham model has a viscosity discontinuity where it changes from a finite value to infinity. This constitutes a phase change where the initially liquid fluid becomes a solid, rendering the fluid governing equations invalid. Although computationally this is not an insurmountable obstacle, it forces modelers to introduce not always well founded ad hoc assumptions for this low shear rate region. In this paper, we propose a $2 \mathrm{D}$ debris flow model based on the shallow water and sediment transport equations and the nonNewtonian Cross rheological formulation. The main peculiarity of the Cross formulation is that it considers that the fluid viscosity changes continuously from a finite value at high shear rates to a very high one (but much less than infinity) as the shear rate tends to zero. Theoretically, the model does not consider a yield stress and therefore the liquid fluid phase is always retained.

In this paper, we expand on the present preliminary results presented by Martinez et al. [9] of the proposed model that aim to test the stability and performance of the model at low shear rates by comparing against experimental data and the analytical solution given by Huang and Garcia [6, 7].

\section{Governing equations}

The model is based on the 2D shallow water equations that describe the free surface flow with the vertically averaged approximation and the sediment continuity equation in Cartesian coordinates:

$$
\begin{gathered}
\frac{\partial H}{\partial t}+\frac{\partial(\bar{u} H)}{\partial x}+\frac{\partial(\bar{v} H)}{\partial y}=0 \\
\frac{\partial(\bar{c} H)}{\partial t}+\frac{\partial(\bar{c} \bar{u} H)}{\partial x}+\frac{\partial(\bar{c} \bar{v} H)}{\partial y}=0
\end{gathered}
$$




$$
\begin{aligned}
& \frac{\partial \bar{u}}{\partial t}+\bar{u} \frac{\partial \bar{u}}{\partial x}+\bar{v} \frac{\partial \bar{u}}{\partial y}+g \frac{\partial \eta}{\partial x}+S_{f x}=0 \\
& \frac{\partial \bar{v}}{\partial t}+\bar{u} \frac{\partial \bar{v}}{\partial x}+\bar{v} \frac{\partial \bar{v}}{\partial y}+g \frac{\partial \eta}{\partial y}+S_{f y}=0
\end{aligned}
$$

where $x$ and $y$ are the horizontal coordinates, $t$ is the time, $\eta$ is the water surface elevation, $H$ is the water depth, $\bar{c}$ is the vertically averaged sediment concentration, $\bar{u}$ and $\bar{v}$ are the vertically averaged velocities in directions $x$ and $y$ respectively, $g$ is the gravitational acceleration and $S_{f x}$ and $S_{f y}$ are the depth integrated stress terms that depend on the rheological model to be used.

\subsection{Implementation of rheological models}

Using the quadratic model postulated by O'Brien and Julien [10]

$$
\tau_{x z}=\tau_{y}+\mu \dot{\gamma}+\zeta \dot{\gamma}^{2}
$$

The first two terms are referred to as the Bingham shear stresses and represent the internal resistance stresses of a Bingham fluid. The last term represents the sum of the dispersive and turbulent shear stresses, which depend on the square of the shear rate.

When the shear stress relationship is depth integrated, it can be rewritten in the following slope form:

$$
S_{f x}=S_{y}+S_{v}+S_{t d}
$$

Where $S_{y}$ is the yield slope, $S_{v}$ is the viscous slope and $S_{t d}$ is the turbulent dispersive slope (O’Brien and Julien [10]). The yield slope is defined as:

$$
S_{y}=\frac{\tau}{\rho g H}
$$

Assuming a vertical parabolic distribution for velocity $u$, the viscous slope is:

$$
S_{v}=\frac{\mu \dot{\gamma}}{\rho g H}=\frac{3 \mu \bar{u}}{\rho g H^{2}}
$$

For the quadratic term, O'Brien and Julien [10] suggest the use of Manning equation, yielding:

$$
S_{t d}=\frac{N^{2} \bar{u}^{2}}{H^{4 / 3}}
$$

The Manning coefficient of roughness $N$, is an empirically derived coefficient, which is dependent on many factors, including bottom surface roughness and sinuosity. 
Finally, for the quadratic rheological model of O'Brien and Julien, the depth integrated stress term is

$$
S_{f x}=\frac{\tau}{\rho g H}+\frac{3 \mu \bar{u}}{\rho g H^{2}}+\frac{N^{2} \bar{u}^{2}}{H^{4 / 3}}
$$

The quadratic model of O'Brian and Julien is combined with the Cross formulation,

$$
\tau_{x z}=\mu_{e f f} \dot{\gamma}+\zeta \dot{\gamma}^{2}
$$

where $\mu_{\text {eff }}$ is the effective viscosity, a continuous variable that changes from a large value at very low shear rates to the fluid dynamic viscosity at higher shear rates. The effective viscosity can be conveniently defined in terms of the Bingham fluid parameters (yield stress and dynamic viscosity) as it is proposed by Shao and Lo [12].

$$
\mu_{\text {eff }}=\frac{\mu_{0}+\mu_{\infty} K_{B} \dot{\gamma}}{1+K_{B} \dot{\gamma}}
$$

with $K_{B}=\frac{\mu_{0}}{\tau_{y}}, \mu_{\infty}=\mu$ and $\mu_{0}=10^{3} \mu$.

Assuming a vertical parabolic distribution for velocity $u, \dot{\gamma}=\frac{3 \bar{u}}{H}$, the depth integrated stress term results in this case

$$
S_{f x}=\frac{\mu_{e f f} \dot{\gamma}}{\rho g H}+\frac{N^{2} \bar{u}^{2}}{H^{4 / 3}}
$$

To determine the viscosity $\mu$ and yield stress $\tau_{y}$ as a function of the volumetric sediment concentration, exponential formulas, as those given by O’Brien and Julien [11], are used:

$$
\begin{gathered}
\mu=\alpha_{1} e^{\beta_{1} \bar{c}} \\
\tau_{y}=\alpha_{2} e^{\beta_{2} \bar{c}}
\end{gathered}
$$

For water-Kaolinite clay mixtures [8], $\alpha_{1}=0.621 \mathrm{E}-3, \beta_{l}=17.3, \alpha_{2}=0.002$ and $\beta_{2}=34.2, \mu$ is in Pa.s, $\tau_{y}$ is in $\mathrm{Pa}$ and $\bar{c}$ is a fraction of 1 .

The shallow water equations and the sediment transport equation are solved by the Galerkin Finite Element method using three-node triangular elements. To solve the system of equations we propose a four-step time stepping scheme and a selective lumping method, as described by Garcia et al. [4]. This scheme 
improves previous finite elements models, allowing larger time steps and enhancing its capability to simulate complex debris flow events without requiring an artificial diffusion term. The use of triangular non structured grids gives the model a great flexibility to accommodate buildings and other obstacles usually present in urbanized alluvial fans.

\section{Results}

The first test problem represents flow from a source of finite size (area A and unit width), a dam break of mud-slide with initial triangular shape (height $\mathrm{H}_{0}$ and length $\mathrm{L}_{0}$ ) on a slope $\theta$, as shown in Fig. 1 . The fluid is a Kaolinite suspension with $\mathrm{Cv}=13.05 \%$ and the flow is considered unsteady, gradually varied, and laminar.

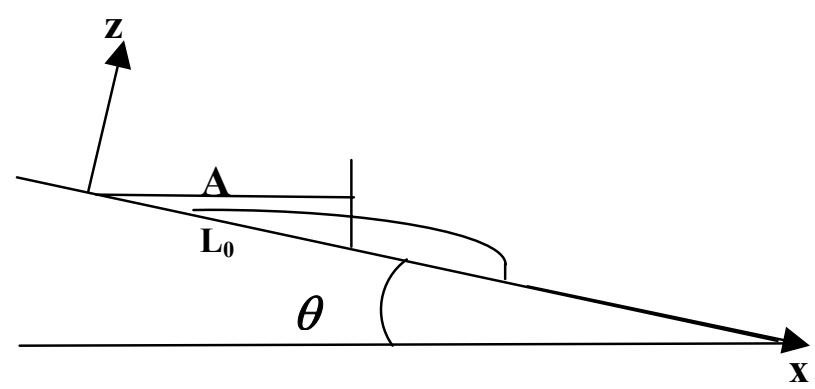

Figure 1: One-dimensional dam break on a incline plane.

The finite element numerical solution is compared with the analytical solution proposed by Huang and Garcia $[6,7]$ and with experimental data given in their work. Although the example is one dimensional, 2D elements are used to discretize the channel using a 2D mesh.

Fig. 2 shows the spreading rate for the following conditions: $\theta=11^{\circ}$, and $\mathrm{A}=24.7 \mathrm{~cm}^{2}\left(\mathrm{~L}_{0}=0.16 \mathrm{~m}\right)$. These results show that the numerical solution using Cross model is in very good agreement with the experimental data for early stages of the solution, where the analytical solution is not as accurate. As time increases, the fluid velocity decreases but the stoppage of the fluid is not totally reached with the numerical solution. After a certain time, the numerical solution tends to increase and deviate from the analytical solution. This is probably caused by the numerical treatment of the dry-wet interface. Ongoing work is addressing this issue.

Fig. 3 shows free surface profiles obtained using Cross formulation. Two different meshes are used, in the new mesh (NM) elements are three times smaller than those in the old mesh (OM). In addition, the selective lumping parameter (related with the finite element solution of the governing equations) is increased from 0.925 to 0.950 . As it is depicted in the figure, the mesh refinement contributes to reduce the numerical diffusion and improves 
substantially the solution in the advancing front. Increasing the selective lumping parameter also enhances the numerical solution, making it closer to the analytical solution.

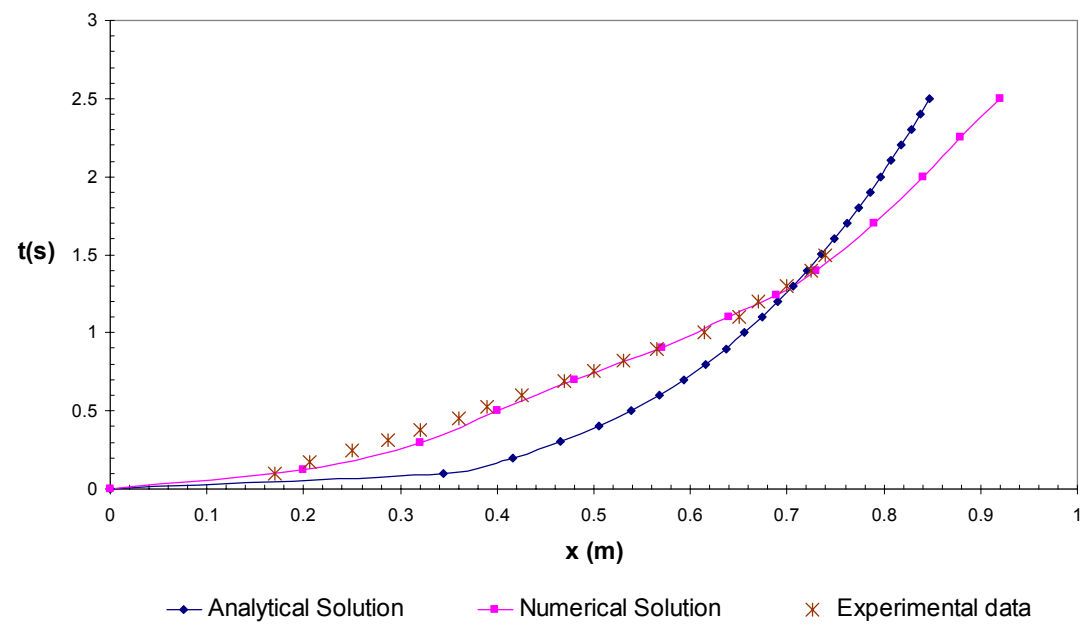

Figure 2: $\quad$ Spreading relation.

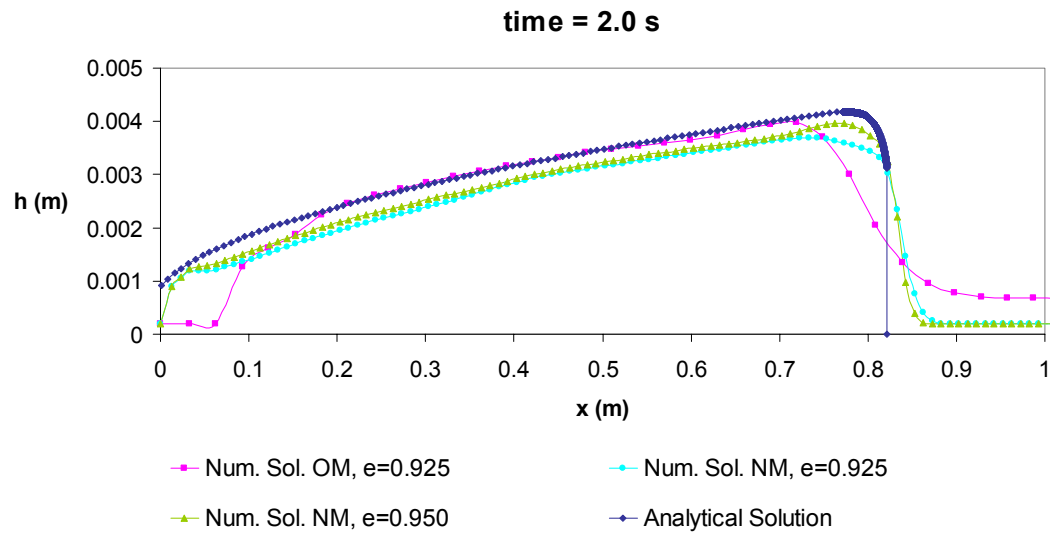

Figure 3: $\quad$ Free surface profiles.

Fig. 4 shows free surface profiles at time $2.3 \mathrm{~s}$ on wet slope, where a layer of fluid exists downstream from the mud-slide. The downstream layer has a height $\mathrm{h} 2$ equals to $0.5 h y$, where $h y$ is the so called yield depth defined by

$$
h y=\frac{\tau_{y}}{\rho g \sin \theta}
$$




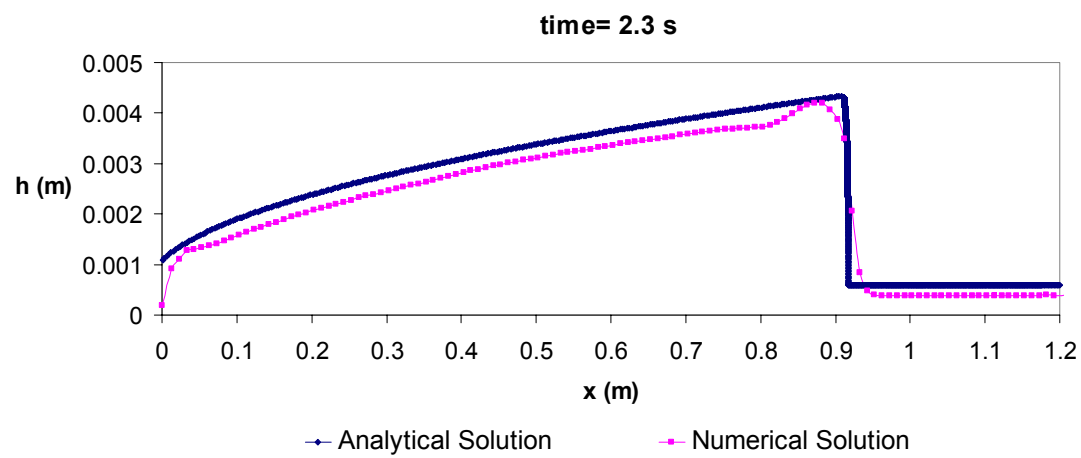

Figure 4: Free surface profiles on wet slope.

The numerical solution obtained using Cross formulation is in very good agreement with the analytical solution [6].

Fig. 5 is a dimensionless plot of the wave shock depth $(h f / H)$ as a function of the shock coordinate $(x f / L)$, where $H$ and $L$ are the initial height and length of the triangular fluid source. The results are for $\lambda=0.04, \lambda=h y / L$, and for different values of $h 2$ [6]. The numerical solution shows an accurate approximation of the analytical solution in all the cases.

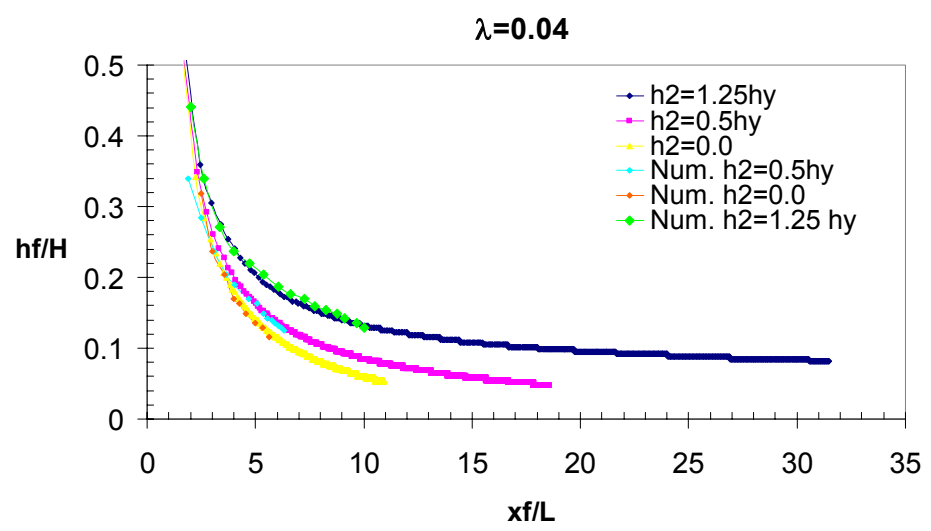

Figure 5: Kinematic-wave shock depth plotted as function of shock coordinate.

Fig. 6 shows the analytical results of a circular dam break on a horizontal plane, presented by Balmforth et al. [1]. The initial condition of the problem is $h^{*}=h / H=1$ for $r^{*}=r / L \leq 1$, where $H$ and $L$ are the initial height and radius of the circular, confined, source of fluid. The dimensionless time $t *$ is defined as

$$
t^{*}=\frac{t L}{V}
$$


where $V$ is a characteristic velocity given by

$$
V=\frac{\rho h H^{3} \cos \theta}{\mu L}
$$

The results are for a Bingham number (dimensionless yield stress) $B=0.15$, with $B$ defined as

$$
B=\frac{\tau_{y} H}{\mu V}
$$

This analytical solution is compared with results given by the Cross formulation (numerical solution) for the same two-dimensional problem. Comparing curves, it is noticeable that the spreading of the numerical solution is larger than the analytical solution at advanced times; however, results are closer at short times.

Preliminary analysis leads us to attribute this behaviour to inaccuracies of the wetting and drying method used in the model, since this factors may play an increasingly important role for smaller depths. We are currently working on further model verifications and testing new wetting and drying algorithms to improve the model accuracy for large times.

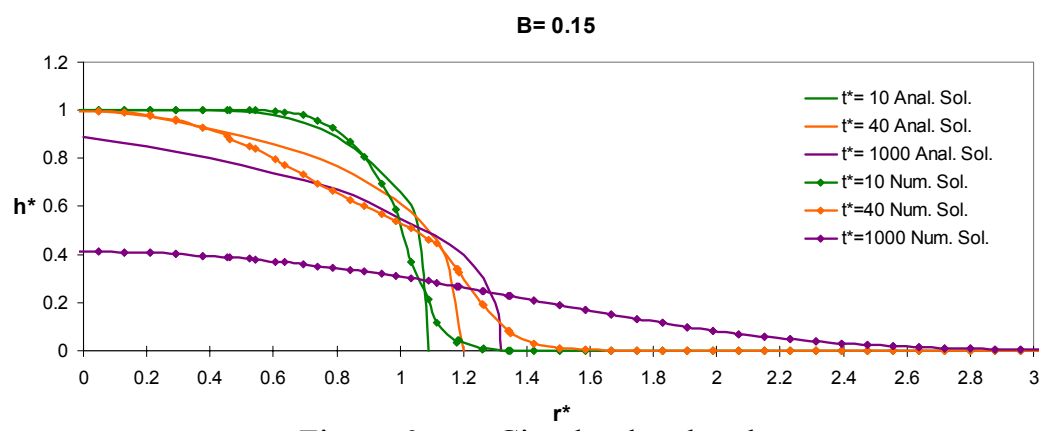

Figure 6: Circular dam break.

\section{Conclusions}

In this paper, we propose a 2D finite element model to simulate debris flows based on the non-Newtonian Cross rheological formulation, where the fluid viscosity changes continuously from a finite value to a very high one (but much less than infinity) as the shear rate tends to zero. The $2 \mathrm{D}$ model is based on the shallow water equations, where the stress terms account for the bottom friction using Manning's formula and the internal friction losses are implemented using the Cross constitutive relations. The numerical method is based on a four-step, selective lumping, explicit time stepping scheme, that solves the system of ordinary differential equations that results from the discretization of the $2 \mathrm{D}$ shallow water equations. We present preliminary tests for one dimensional and two dimensional dam break problems, comparing the model results with some experimental data and analytical solutions. Model results show very good agreement with the 
experimental data and analytical solutions in one dimensional cases. For the flow early stages, the numerical solution agrees better with the experimental data than with the analytical solution. However; in final stages, the numerical solution predicts that the fluid front advances more rapidly than the analytical solution and measured fluid front. The same results are observed in a 2D problem; however, experimental data to compare with is scarce. The proposed 2D debris flow model using Cross rheological formulation provides very stable results even in the range of very low shear rates, where other discontinuous constitutive relations that use the yield stress may become unstable.

\section{References}

[1] Balmforth, N. J., Craster, R. V., Rust, A.C. \& Sassi, R. Viscoplastic flow over inclined surface. Journal of Non-Newtonian Fluid Mechanics, 139:103-127, 2006.

[2] Barnes, H.A., Hutton J.F. \& Walters, K. An introduction to rheology, Elsevier: Amsterdam, 1989.

[3] Coussot, P. Mudflow rheology and dynamics. IAHR Monograph Series, Balkema, 1997.

[4] García-Martínez, R. Espinoza, E. Valera \& M. González. An explicit twodimensional finite element model to simulate short and long term bed evolution in alluvial rivers. Journal of Hydraulic Research, 44 (6): 2006.

[5] Hammad, K. \& Vradis, G.C. Flow of a non-Newtonian Bingham plastic through an axisymmetric sudden contraction: effects of Reynolds and yield numbers. Numerical Methods Non- Newtonian Fluid Dynamics, ASME 179:63-90, 1994.

[6] Huang, X. \& Garcia, M.H. Asymptotic solution for Bingham debris flows. Debris Flow Hazards Mitigation, ASCE, New York, 561-575, 1997.

[7] Huang, X. \& Garcia, M.H. A Herschel-Bulkley model for mud flow down a slope. Journal of Fluid Mechanics, U. K., 374:305-333, 1998.

[8] Komatina, D. \& Jomanovic, M. Experimental study of steady and unsteady free surface flows with water-clay mixtures. Journal of Hydraulic Research, 35:579-590, 1997.

[9] Martinez, C., F. Miralles-Wilhem and R. Garcia-Martinez. A 2D finite element debris flow model based on the cross rheology formulation. Fourth International Conference on Debris-Flow Hazards Mitigation: Mechanics, Prediction and Assessment. Chendu, China, September, 2007.

[10] O'Brien, J.S. and Julien, P.Y. Physical properties and mechanics of hyperconcentrated sediment flows. ASCE Specialty Conference on the Delineation of Landslides, Floods and Debris Flows Hazards. ASCE, New York, 260-279, 1985.

[11] O'Brien, J.S. \& Julien, P.Y. Laboratory analysis of mudflows properties. Journal of Hydraulic Engineering, ASCE 114:877-887, 1988.

[12] Shao, S. \& Lo, E.Y. Incompressible SPH method for simulating Newtonian and Non-Newtonian flows with a free surface. Advances in Water Resources, 26:787-800, 2003. 